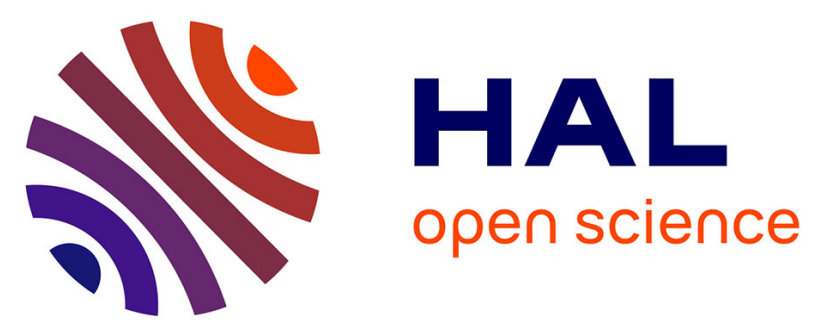

\title{
COMBINING PATCH-BASED ESTIMATION AND TOTAL VARIATION REGULARIZATION FOR 3D INSAR RECONSTRUCTION
}

Charles-Alban Deledalle, Loïc Denis, Giampaolo Ferraioli, Florence Tupin

\section{- To cite this version:}

Charles-Alban Deledalle, Loïc Denis, Giampaolo Ferraioli, Florence Tupin. COMBINING PATCHBASED ESTIMATION AND TOTAL VARIATION REGULARIZATION FOR 3D INSAR RECONSTRUCTION. Geoscience and Remote Sensing Symposium (IGARSS), Jul 2015, Milan, Italy. ujm01219134

\section{HAL Id: ujm-01219134}

\section{https://hal-ujm.archives-ouvertes.fr/ujm-01219134}

Submitted on 22 Oct 2015

HAL is a multi-disciplinary open access archive for the deposit and dissemination of scientific research documents, whether they are published or not. The documents may come from teaching and research institutions in France or abroad, or from public or private research centers.
L'archive ouverte pluridisciplinaire $\mathbf{H A L}$, est destinée au dépôt et à la diffusion de documents scientifiques de niveau recherche, publiés ou non, émanant des établissements d'enseignement et de recherche français ou étrangers, des laboratoires publics ou privés. 


\title{
COMBINING PATCH-BASED ESTIMATION AND TOTAL VARIATION REGULARIZATION FOR 3D INSAR RECONSTRUCTION
}

\author{
Charles-Alban Deledalle ${ }^{(1)}$, Loic Denis ${ }^{(2)}$, Giampaolo Ferraioli ${ }^{(3)}$, Florence Tupin $^{(4)}$ \\ ${ }^{(1)}$ CNRS-Univ. Bordeaux, IMB - Talence, France \\ ${ }^{2}$ CNRS-Univ. Saint-Etienne, Laboratoire Hubert Curien - Saint-Etienne, France \\ ${ }^{(3)}$ Dipartimento di Scienze e Tecnologie \\ Università degli Studi di Napoli "Parthenope" - Naples, Italy \\ ${ }^{(4)}$ Institut Mines-Télécom, Télécom ParisTech CNRS-LTCI Paris, France
}

\begin{abstract}
In this paper we propose a new approach for height retrieval using multi-channel SAR interferometry. It combines patchbased estimation and total variation regularization to provide a regularized height estimate. The non-local likelihood term adaptation relies on NL-SAR method, and the global optimization is realized through graph-cut minimization. The method is evaluated both with synthetic and real experiments.
\end{abstract}

Index Terms - SAR interferometry, multi-channel InSAR, Non-local means, TV regularization

\section{INTRODUCTION}

High accuracy Digital Elevation Model of the observed ground scene can be generated using Interferometric Synthetic Aperture Radar systems in a multi-channel configuration [1, 2]. An effective way to combine the available multi-channel interferometric data is to exploit statistical estimation methods [3, 4]. The height retrieval from InSAR data poses at least two difficulties: (i) the phase information is very noisy, hence it requires some smoothing while preserving at best the spatial resolution; and (ii) the phase is wrapped, so that it may not be uniquely invertible to provide the height.

In this paper we propose to adapt patch-based methods, in particular NL-SAR approach [5], to recover directly an elevation exploiting the statistical distribution of data in the interferometric multi-channel case. Often, for simplicity, the statistical independence approximation of the multi-channel interferometric phases is adopted for describing the statistical distribution of the data. In a recently presented paper [6], it has been shown that the height reconstruction can be effectively obtained exploiting the whole complex data acquired in the multi-baselines interferometric configuration, removing the statistical independence assumption. Following this approach, in this paper rather than marginalizing the data distribution with respect to missing parameters, as is usually done in the literature, we exploit the joint distribution as done in [6].

The adopted strategy includes two steps: first NL-SAR method can provide non-local (i.e., patch-based) estimates of the covariance matrices at each pixel from the multichannel available images, while preserving at best the resolution. Second, height values can be regularized using an edge-preserving regularization such as total variation [7]. Following the approach proposed in [8] the problem can be formulated as maximum a posteriori estimation with a modified likelihood term. The modified likelihood enforces that the solution at pixel $i$ is close to the observed value at that pixel but also to the observed values at pixels $j$ that are similar (i.e., whose surrounding patches are in good match). Spatial regularity is enforced through the total variation term.

\section{THE MODEL}

Let us consider a pixel $i$ of the whole image and let us suppose that $D$ different channels are available. The measured values can be collected in a vector $\boldsymbol{g}_{i}$ of $D$ complex values. This vector is generally called the scattering vector. Under the classical hypothesis of fully developed speckle (Goodman's model), the observations $\boldsymbol{g}_{i}$ at pixel $i$ are distributed according to a circular complex Gaussian:

$$
\mathrm{p}\left(\boldsymbol{g}_{i} \mid \boldsymbol{\Sigma}_{i}\right)=\frac{1}{\pi^{D} \operatorname{det}\left(\boldsymbol{\Sigma}_{i}\right)} \exp \left(-\boldsymbol{g}_{i}^{\dagger} \boldsymbol{\Sigma}_{i}^{-1} \boldsymbol{g}_{i}\right)
$$

with $\boldsymbol{g}_{i}^{\dagger}$ the Hermitian transpose of column vector $\boldsymbol{g}_{i}$. This distribution relies on the complex covariance matrix $\boldsymbol{\Sigma}_{i}$ at pixel $i$ which depends on the radiometry $R$, the inter-channel coherence $\gamma_{a, b}$ and the interferometric phases $\psi_{a, b}$ :

$$
\boldsymbol{\Sigma}=R\left(\begin{array}{cccc}
1 & s_{1,2} & \cdots & s_{1, D} \\
s_{1,2} & 1 & & s_{2, D} \\
\vdots & & \ddots & \vdots \\
s_{1, D} & s_{2, D} & & 1
\end{array}\right)
$$


where the generic element of the matrix is defined as $s_{a, b}=$ $\gamma_{a, b} \exp \left(j \psi_{a, b}\right)$. The interferometric phases $\psi_{a, b}$ are related to the height $h$ through a function $f_{a, b}$ that accounts for the interferometric baseline, eventual atmospheric distortions and other calibration parameters: $\psi_{a, b}=f_{a, b}(h)$ [2]. In Eq. (2) the radiometry $R$ is considered the same among the available channels.

In order to estimate the height of the pixels, a Maximum a Posteriori (MAP) approach is implemented. The likelihood term of the a posteriori distribution is derived from the distribution of Eq. (1) using a non-local approach: the data that can help in the estimation of the height $\hat{h}_{i}$ at pixel $i$, are not only the observed value at that pixel but also the observed values at pixels $j$ that have similar surroundings. This leads to a weighted maximum likelihood estimation [9]. Concerning the $a$ priori term the Total Variation (TV) model is adopted. According to Bayesian approach and taking the logarithm of the non-local likelihood and the a priori term, the estimation problem becomes:

$$
\hat{\boldsymbol{h}}^{(\mathrm{NL})}=\underset{\boldsymbol{h}}{\arg \min }-\sum_{i} \sum_{j} \omega_{i, j} \log \mathrm{p}\left(\boldsymbol{g}_{j} \mid h_{i}\right)+\mathrm{TV}(\boldsymbol{h})
$$

where $\boldsymbol{h}$ is the vector of unknown heights to be estimated (one height $h_{i}$ per pixel), $\omega_{i, j}$ are the non-local coefficients that balance the weight of observations $\boldsymbol{g}_{j}$ at pixel $j$ according to their relevance for the estimation $h_{i}$ at pixel $i$ and $\operatorname{TV}(\boldsymbol{h})$ is the total variation energy defined as $\operatorname{TV}(\boldsymbol{h})=\sum_{(i, j)}\left|h_{i}-h_{j}\right|$ where $(i, j)$ span all neighbor pixels.

The function of Eq. (3) can be simplified into an expression with a separable data-term. Let us consider only the first part (non-local likelihood term). After dropping the constant $D \log \pi$ which is irrelevant for the optimization problem, the non-local negative log-likelihood can be written as:

$$
\begin{aligned}
& -\sum_{j} \omega_{i, j} \log \mathrm{p}\left(\boldsymbol{g}_{j} \mid \boldsymbol{\Sigma}_{i}\right) \propto \\
& \sum_{j} \omega_{i, j}\left[\boldsymbol{g}_{j}^{\dagger} \boldsymbol{\Sigma}_{i}^{-1} \boldsymbol{g}_{j}+\log \operatorname{det}\left(\boldsymbol{\Sigma}_{i}\right)\right] .
\end{aligned}
$$

By introducing the coefficients $\lambda_{i}^{(\mathrm{NL})}=\sum_{j} \omega_{i, j}$ and the nonlocal empirical covariance matrix $\boldsymbol{C}_{i}^{(\mathrm{NL})}=\frac{1}{\lambda_{i}} \sum_{j} \omega_{i, j} \boldsymbol{g}_{j} \boldsymbol{g}_{j}^{\dagger}$, the non-local negative log-likelihood becomes:

$$
\begin{aligned}
& -\sum_{j} \omega_{i, j} \log \mathrm{p}\left(\boldsymbol{g}_{j} \mid \boldsymbol{\Sigma}_{i}\right) \propto \\
& \lambda_{i}^{(\mathrm{NL})} \log \operatorname{det}\left(\boldsymbol{\Sigma}_{i}\right)+\lambda_{i}^{(\mathrm{NL})} \operatorname{tr}\left(\boldsymbol{\Sigma}_{i}^{-1} \boldsymbol{C}_{i}^{(\mathrm{NL})}\right) .
\end{aligned}
$$

Finally, since $\log \operatorname{det}\left(\boldsymbol{\Sigma}_{i}\right)$ is independent from $h_{i}$ [6], and by adding the a priori term, the MAP solution for the height estimation is given by:

$$
\hat{\boldsymbol{h}}^{(\mathrm{NL})}=\underset{\boldsymbol{h}}{\arg \min }-\sum_{i} \lambda_{i}^{(\mathrm{NL})} \operatorname{tr}\left(\boldsymbol{\Sigma}_{i}^{-1} \boldsymbol{C}_{i}^{(\mathrm{NL})}\right)+\mathrm{TV}(\boldsymbol{h})
$$

Thus, weighting the likelihood term with non local weights is equivalent of computing a non local estimation of the observations and injecting it in the energy.

The non-local empirical covariance matrix $C_{i}^{(\mathrm{NL})}$ can be computed using NL-SAR method [5]. NL-SAR is a patchbased approach adapted to multi-channel complex SAR data. Patch similarity is derived from a generalized likelihood ratio test taking the circular complex Gaussian distribution. A set of parameters (size of the patch, size of the search window, strength of pre-filtering) is tested, and the results are combined after a debiasing step to provide the "best" estimate (the quality being given by the equivalent number of looks).

The sum of non-local weights $\lambda_{i}^{(\mathrm{NL})}=\sum_{j} \omega_{i, j}$ could be set to any positive values (the weighted mean being invariant up to a multiplication factor). However, it should differ from 1 in order to give more importance to observed values $\boldsymbol{g}_{j}$ when many locations $j$ are similar to the reference location $i$ compared to the case of a pixel $i$ with no similar pixel within the search window. Following the development of [8], we may define $\lambda_{i}^{(\mathrm{NL})}$ to be the inverse of the estimated residual standard deviation: $\lambda_{i}=\sqrt{\hat{L}_{i}^{\mathrm{NLRB}}}$, with $\hat{L}_{i}^{\mathrm{NLRB}}$ defined as the estimated equivalent number of looks at pixel $i$ also provided by NL-SAR method [5].

Concerning the optimization step, due to the non-convexity of the data fidelity term involved in (6), a graph-cut based minimization method has been adopted. The minimization problem involves a non-convex but separable data-term and a convex pairwise term. It is solved exactly (up to the chosen height quantization) by graph-cuts using Ishikawa's construction [10].

\section{NUMERICAL EXPERIMENTS}

In order to validate the presented approach a simulated test case has been considered. The dataset is made of three complex images acquired with different baselines. Two of the three available interferograms are shown in the first row of Figure 1, together with the mean coherence map and the true profile. The estimation of the true profile is not an easy task due to the presence of noise and of high phase jumps. The second row shows (from left to right) the results obtained using the Maximum Likelihood (ML) approach proposed in [6], using the ML-NL approach (using only the likelihood term of Eq. (6)), the MAP-NL approach (using Eq. (6) and a sub-optimal minimization procedure based on Iterated Conditional Modes (ICM)) and the MAP-NL approach (using Eq. (6) and an optimal minimization procedure based on graphcuts [10].) MAP-NL achieves a strong reduction of the variance of height estimation while preserving edges (no blurring phenomenon).

The presented approach has also been tested on a real dataset. This dataset is composed of three COSMO-SkyMed Stripmap images acquired close to Naples train station. Two 

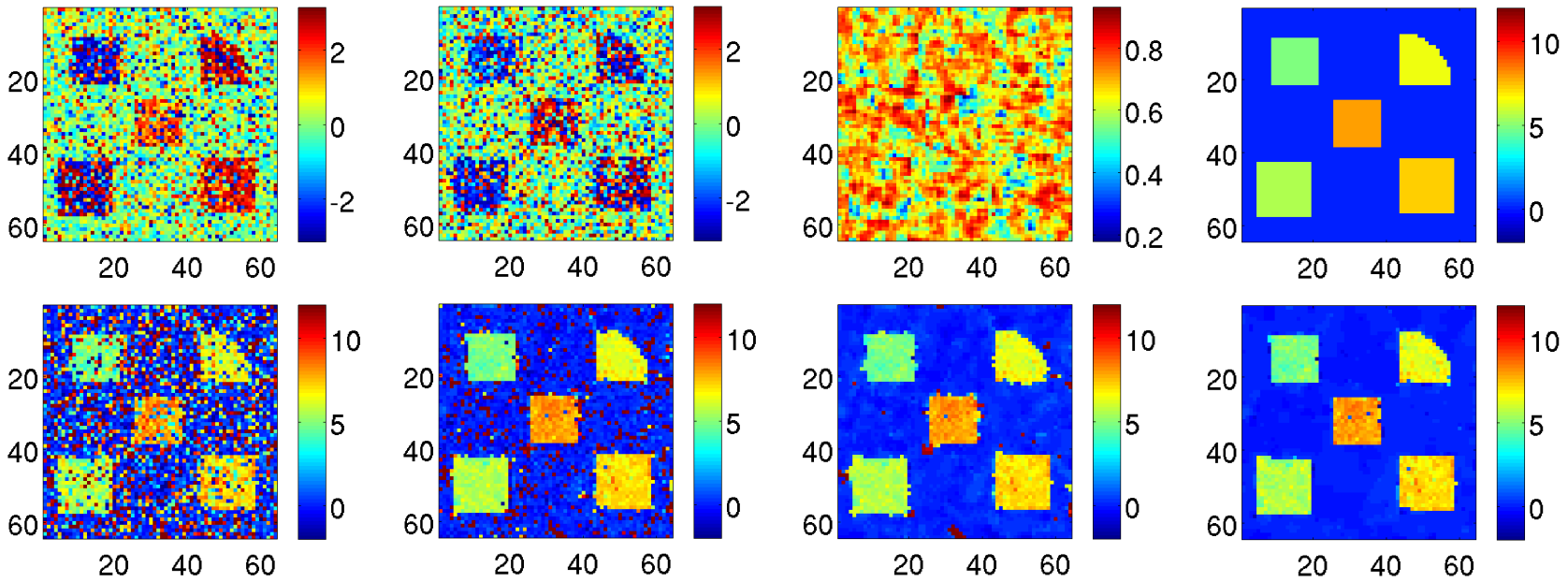

Fig. 1. Simulated Dataset. First row: Two of the three available interferograms, mean coherence map and the true profile. Second row: the results using the ML corrlelated approach, the ML-NL correlated approach, the MAP-NL approach based on ICM and the MAP-NL approach based on graph cuts

of the three available interferograms are shown in the first row of Figure 2, together with the logarithm of the mean amplitude and the mean coherence map. The second row shows (from left to right) the results obtained using the Maximum Likelihood (ML) approach proposed in [6], using the MLNL approach (using only the likelihood term of Eq. (6)), the MAP-NL approach (using Eq. (6) and a sub-optimal minimization procedure based on ICM) and the MAP-NL approach (using Eq. (6) and an optimal minimization procedure based on graph-cuts [10]). Independent estimation of the height at each pixel leads to a very noisy result (i.e., strong variance of the heights). The regularization reduces these fluctuations without noticeable resolution loss.

\section{CONCLUSIONS}

In this paper, a non-local regularized method of height computation for multi-channel InSAR interferometry has been proposed. First results are promising and further work includes the following points. First, other experiments have to be led, specially on the influence of the coherence values on the results. Besides, the impact of channel number vs. the coherence values will be studied. On the methodological part, the influence of reflectivity and coherence values (which are pre-estimated in the presented approach) has to be analyzed and a joint estimation scheme should be studied.

\section{REFERENCES}

[1] R. Bamler and P. Hartl, "Syntetic aperture radar interferometry," Inverse Problem, vol. 14, pp. R1-R54, August 1998.
[2] G. Fornaro and V. Pascazio, "SAR Interferometry and Tomography: Theory and Applications," Academic Press Library in Signal Processing, Elsevier, 2013.

[3] M. Eineder and N. Adam, "A maximum-likelihood estimator to simultaneously unwrap, geocode, and fuse SAR interferograms from different viewing geometries into one digital elevation model," Geoscience and Remote Sensing, IEEE Transactions on, vol. 43, no. 1, pp. 24-36, 2005.

[4] G. Ferraiuolo, V. Pascazio, and G. Schirinzi, "Maximum a posteriori estimation of height profiles in InSAR imaging," Geoscience and Remote Sensing Letters, IEEE, vol. 1, no. 2, pp. 66-70, 2004.

[5] C.-A Deledalle, L. Denis, F. Tupin, A Reigber, and M. Jager, "NL-SAR: A Unified Nonlocal Framework for Resolution-Preserving (Pol)(In)SAR Denoising," Geoscience and Remote Sensing, IEEE Transactions on, vol. PP, no. 99, pp. 1-18, 2014.

[6] Fabio Baselice, Alessandra Budillon, Giampaolo Ferraioli, Vito Pascazio, and Gilda Schirinzi, "Multibaseline SAR Interferometry from Complex Data," Selected Topics in Applied Earth Observations and Remote Sensing, IEEE Journal of, vol. 7, no. 7, pp. 2911-2918, 2014.

[7] Aymen Shabou, Fabio Baselice, and Giampaolo Ferraioli, "Urban digital elevation model reconstruction using very high resolution multichannel InSAR data," Geoscience and Remote Sensing, IEEE Transactions on, vol. 50, no. 11, pp. 4748-4758, 2012. 

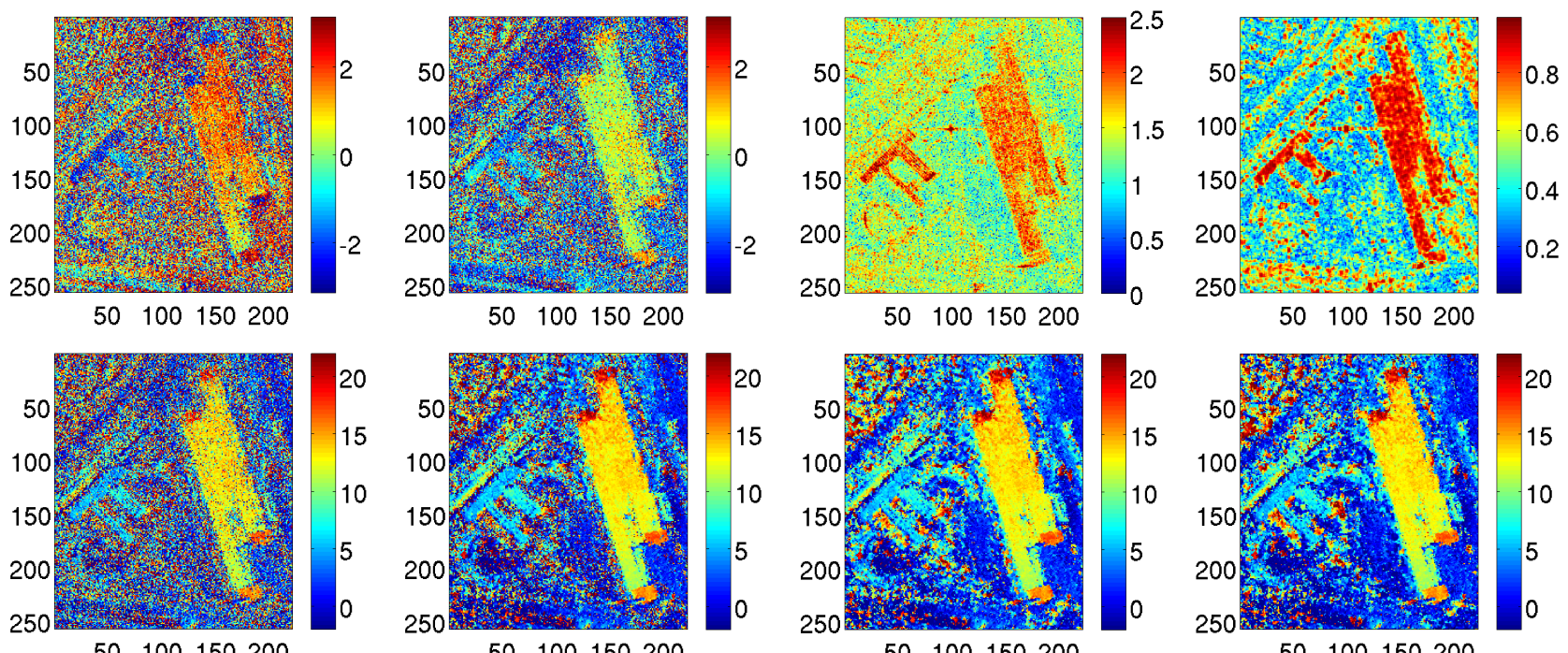

Fig. 2. Real Dataset. First row: Two of the three available interferograms, logarithm of the mean amplitude image and the mean coherence map. Second row: the results using the ML correlated approach, the ML-NL correlated approach, the MAP-NL approach based on ICM and the MAP-NL approach based on graph cuts

[8] C. Sutour, C.-A Deledalle, and J.-F. Aujol, "Adaptive Regularization of the NL-Means: Application to Image and Video Denoising," Image Processing, IEEE Transactions on, vol. 23, no. 8, pp. 3506-3521, Aug 2014.

[9] C.-A. Deledalle, L. Denis, and F. Tupin, "Iterative weighted maximum likelihood denoising with probabilistic patch-based weights," Image Processing, IEEE Transactions on, vol. 18, no. 12, pp. 2661-2672, Dec 2009.

[10] Hiroshi Ishikawa, "Exact optimization for markov random fields with convex priors," Pattern Analysis and Machine Intelligence, IEEE Transactions on, vol. 25, no. 10, pp. 1333-1336, 2003. 